\title{
Linfadenectomía mediastinal para el cáncer de esófago mediante un abordaje miniinvasivo. Diferencias entre en el adenocarcinoma y el carcinoma escamoso
}

\author{
Norberto Daniel Velasco Hernández, ${ }^{1}$ Héctor Raúl Horiuchi, ${ }^{2}$ Lucas Alberto Rivaletto, ${ }^{3}$ María \\ Micaela Zicavo, ${ }^{4}$ Lucila Fernández ${ }^{5}$ \\ ${ }^{1}$ Jefe de Consultorio Externo del Servicio de Cirugía General del H.I.G.A San Martín de La Plata. Cirujano del Area de Cirugía esófago-gástrica del \\ Hospital Italiano de La Plata e Instituto del Diagnóstico de la ciudad de La Plata. \\ ${ }^{2}$ Jefe de Sala del Servicio de Cirugía General del H.I.G.A San Martín de La Plata. Jefe del Área de Cirugía esófago-gástrica del Hospital Italiano de \\ La Plata y cirujano del Instituto del Diagnóstico de la ciudad de La Plata. \\ ${ }^{3}$ Cirujano de planta del Servicio de Cirugia General del H.I. G.A San Martín de La Plata. Cirujano del Área de Cirugía esófago-gástrica del Hospital \\ Italiano de La Plata e Instituto del Diagnóstico de la ciudad de La Plata. \\ ${ }^{4}$ Médica del Servicio de Oncología del H.I.G.A San Martín de La Plata e Instituto del Diagnóstico de la ciudad de La Plata. \\ ${ }^{5}$ Médica residente del Servicio de Cirugía General del H.I.G.A San Martín de La Plata.
}

Hospital Interzonal General de Agudos General San Martín, Instituto de Diagnóstico y Hospital Italiano de la ciudad de La Plata. Universidad Nacional de La Plata. Buenos Aires, Argentina.

Acta Gastroenterol Latinoam 2020;50(2):133-139

Recibido: 23/10/2019 / Aceptado: 20/01/2020 / Publicado online: 29/06/2020

\section{Resumen}

Antecedentes. En la era del tratamiento multimodal, la cirugía continúa siendo el pilar principal para el cáncer de esófago. El tipo de linfadenectomía plantea controversias importantes entre los paises de Oriente y Occidente, principalmente por la falta de consenso en la extensión de la misma. Objetivos. Analizar el tipo de linfadenectomía mediastinal realizada para el adenocarcinoma y carcinoma escamoso, de

Correspondencia: Daniel $N$ Velasco Hernández $8 N^{\circ} 2877$ CP: 1923. Berisso, Buenos Aires, Argentina Cel: 542214349596

Correo electrónico: daniels84@hotmail.com un grupo de pacientes a los que se le efectuó esofagectomía miniinvasiva en posición prona. Material y métodos. En el periodo comprendido entre noviembre de 2011 y agosto de 2019 fueron intervenidos quirúrgicamente 51 pacientes por cáncer de esófago potencialmente curable, a los cuales se les efectuó esofagectomía miniinvasiva en posición prona con linfadenectomía de dos campos (mediastinal y abdominal). Sobre la base del tipo histológico y localización del tumor se efectuaron los diferentes tipos de vaciamientos. Resultados. La mediana en el número de ganglios obtenidos para el adenocarcinoma fue de 5,0 (3-16), mientras que para los casos de carcinoma escamoso fue de 8,0 (4-38) ( $p=0,019)$. Se registraron dos complicaciones intraoperatorias: una lesión del bronquio fuente izquierdo durante la linfadenectomía subcarinal, y una lesión del nervio recurrente derecho durante una linfadenectomía extendida. Conclusión. Podemos afirmar que la linfadenectomía mediastinal es un procedimiento factible de realizar con un abordaje miniinvasivo en posición prona, por ofrecer una adecuada visualización de las estructuras anatómicas y la obtención de un número 
aceptable de ganglios. Sin embargo, está supeditada a complicaciones que, en algunos casos, pueden ser graves.

Palabras claves. Cáncer de esófago, linfadenectomía mediastinal, esofagectomía miniinvasiva.

\section{Mediastinal Iymphadenectomy for oesophageal cancer by minimally invasive approach. Differences between adenocarcinoma and squamous carcinoma}

\section{Summary}

Background. In the multimodal treatment era, surgery remains the mainstay for oesophageal cancer. The type of lymphadenectomy raises important controversies between eastern and western countries, mainly due to the lack of consensus on its extension. Objectives. Analyse the type of mediastinal lymphadenectomy performed for adenocarcinoma and squamous cells carcinoma, of a group of patients who underwent a prone position minimally invasive esophagectomy. Material and methods. From November 2011 to August 2019, 51 patients were treated for potentially curable oesophageal cancer who underwent minimally invasive esophagectomy with two-field lymphadenectomy (mediastinal and abdominal). Based on the histological type and location of the tumour, the different types of lymph nodes dissection were performed. Results. The median of nodes obtained for Adenocarcinoma was 5.0 (3-16) while for squamous cells carcinoma cases it was $8.0(4-38)(p=0.019)$. Two intraoperative complications were recorded: a lesion of the left source bronchus during sub carinal lymphadenectomy, and injury to the right recurrent nerve during an extended lymphadenectomy. Conclusion. We can conclude the mediastinal lymphadenectomy is a feasible procedure to perform with a minimally invasive approach in prone position, for offering an adequate visualization of the anatomical structures and obtaining an acceptable number of nodes. However, it is subject to complications, some of which could be serious.

Key words. Oesophageal cancer, mediastinal lymphadenectomy, minimally invasive esophagectomy.

\section{Introducción}

En la era del tratamiento multimodal, la cirugía continúa siendo el pilar principal para el cáncer de esófago. En la actualidad el tipo de linfadenectomía (LN) plantea controversias importantes entre países de Oriente y
Occidente, principalmente por la falta de consenso en la extensión de la misma. ${ }^{1} \mathrm{Si}$ bien en casi todo el mundo se realizan vaciamientos ganglionares de dos campos (abdominal y torácico), en algunos centros de Asia, fundamentalmente del Japón, se efectúan vaciamientos de tres campos (abdominal, torácico y cervical). ${ }^{2}$ A su vez, no existe un criterio uniforme para establecer cuál es la LN más apropiada solo para el compartimiento mediastinal. Estas discrepancias se deben a diferencias en el tipo histológico y en la localización del tumor; en los países asiáticos alrededor del $95 \%$ son carcinomas escamosos (CES) y de ubicación en el esófago torácico, mientras que en los países occidentales más del $80 \%$ de los casos son adenocarcinomas (AC) localizados en esófago distal. ${ }^{3-5}$

Desde las primeras publicaciones por Dallamagne y Cuschieri de esofagectomías realizadas por vía miniinvasiva, estas se efectúan cada vez con más frecuencia en el mundo. ${ }^{6}$ La magnificación de la imagen y la posición del paciente permiten una observación con mejor claridad de las estructuras anatómicas del mediastino y, por lo tanto, permiten realizar una apropiada LN con obtención de un adecuado número de ganglios. ${ }^{6-8}$

El objetivo de este trabajo es analizar el tipo de linfadenectomía mediastinal realizada para el AC y CES, en un grupo de pacientes a los que se les efectuó esofagectomía miniinvasiva en posición prona (EMI PP).

\section{Material y métodos}

En el período comprendido entre noviembre de 2011 y agosto de 2019 fueron intervenidos quirúrgicamente 51 pacientes por cáncer de esófago potencialmente curable, a los cuales se les efectuó EMI PP con LN de dos campos. El procedimiento fue realizado por el mismo equipo quirúrgico en los siguientes centros: Hospital Interzonal General de Agudos General San Martín, Instituto de Diagnóstico y Hospital Italiano de la ciudad de La Plata. El estudio es observacional de corte transversal.

Técnica: La cirugía realizada en 46 casos fue mediante triple abordaje (procedimiento de Mckeown) y solo en 5 casos de adenocarcinoma de esófago inferior se realizó un procedimiento de Ivor Lewis (abordaje abdominal y torácico). Las características de los pacientes y el tipo de tratamiento se muestran en la Tabla $\mathrm{N}^{\circ} 1$. Durante el tiempo torácico, el paciente siempre se ubicó en decúbito ventral con 3 puertos, como inicialmente se describió la técnica de la EMI en PP, aunque ocasionalmente se recurrió a un 4to trócar. ${ }^{7,-11}$

En la Figura 1 se muestran las estructuras anatómicas y los diferentes grupos ganglionares desde una visión en decúbito prono. 
Tabla 1. Características de los pacientes.

\begin{tabular}{lr}
\hline Características & Datos (\%) \\
\hline Edad & \\
Media & $58,7 \pm 9,8$ \\
Sexo & \\
Femenino & $16(31,4)$ \\
Masculino & $35(38,6)$ \\
Tipo histológico & \\
Adenocarcinoma & $33(64,7)$ \\
Carcinoma epidermoide & $18(35,3)$ \\
Estadios & \\
I & $3(5,9)$ \\
II & 9 \\
(17,6) & \\
IIA & $4(7,8)$ \\
IIB & $19(37,3)$ \\
III & $14(27,5)$ \\
IVA & $2(3,9)$ \\
Neoadyuvancia & \\
Sí & $22(43,1)$ \\
No & $29(56,9)$, \\
Cirugía & \\
McKeown & \\
Ivor Lewis & 46 \\
\hline
\end{tabular}

Figura 1. Visión en decúbito prono del mediastino posterior. Nódulos linfáticos según la nomenclatura de American Joint Comittee on Cancer (AJCC 8 th). ${ }^{12}$

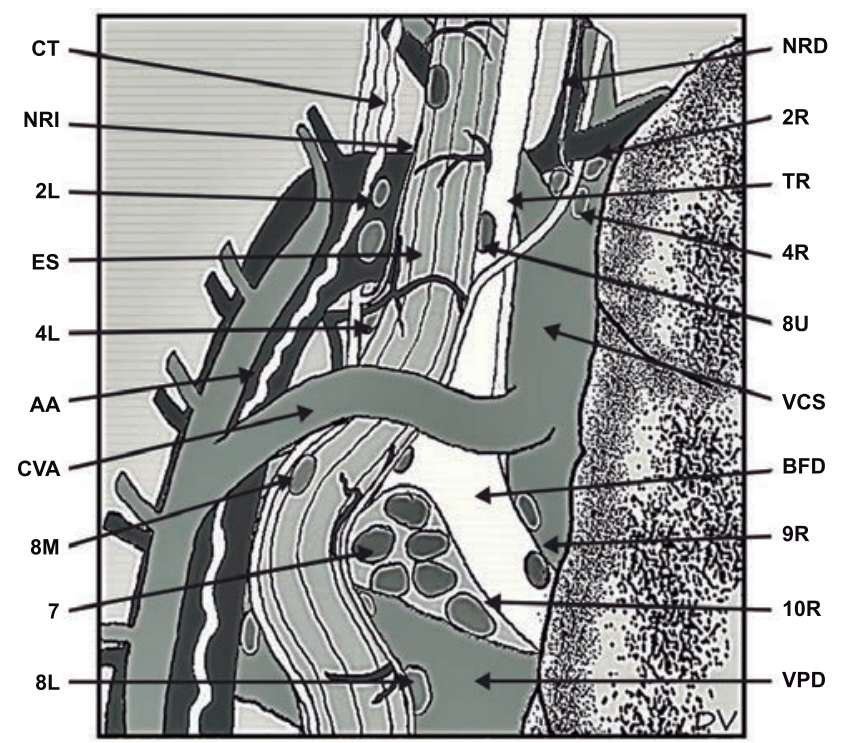

CT: conducto torácico, NRD: nervio recurrente derecho, NRI: nervio recurrente izquierdo, 2R: ganglios paratraqueales superiores derechos, 2L: ganglios paratraqueales superiores izquierdos, TR: tráquea, ES: esófago, 4R: ganglios paratraqueales inferiores derechos, 4L: ganglios paratraqueales inferiores izquierdos, 8U: ganglios paraesofágicos torácicos superiores, AA: arteria aorta, VCS: vena cava superior, CVA: cayado de vena ácigos mayor, BFD: bronquio fuente derecho, 8M: ganglios paraesofágicos torácicos medios, 9: ganglios del ligamento pulmonar derecho, 7: ganglios subcarinales, VPD: vena pulmonar derecha, 8L: ganglios paraesofágicos torácicos inferiores, 10R: ganglios traqueo-bronquiales derechos.

\section{Extensión de la linfadenectomía.}

Se utilizó la clasificación de la International Society for Diseases of the Esophagus (ISDE) del año 1994 (actualizada en 2003) que la divide en: tipo I o estándar, que comprende hasta los ganglios subcarinales; tipo II o extendida, aquella que realiza el vaciamiento hasta el espacio paratraqueal derecho; tipo IIl, linfadenectomía mediastinal total de ambos espacios paratraqueales y de los nervios recurrenciales, y tipo IV, que es la linfadenectomía de tres campos: abdominal, mediastinal y cervical. ${ }^{2,13}$ En la Figura 2 se señalan las diferencias en la extensión de la linfadenectomía mediastinal desde una posición en decúbito prono.

Sobre la base del tipo histológico y la localización del tumor se efectuaron los diferentes tipos de vaciamientos. Se utilizó la división de la AJCC, que considera la distancia desde la arcada dentaria superior: de 15 a $18 \mathrm{~cm}$, esófago cervical; de 18 a 24 cm esófago torácico superior; de 24 a $32 \mathrm{~cm}$ esófago torácico medio, y de 32 a $40 \mathrm{~cm}$ esófago torácico inferior e intraabdominal.

En todos los adenocarcinomas y en un caso de carcinoma epidermoide de localización en esófago inferior se efectuaron vaciamientos del tipo estándar.

En los casos de carcinomas escamosos de ubicación torácica media se realizó linfadenectomía extendida, y en los de localización en esófago torácico superior LN total $^{12,14}$ La neoadyuvancia empleada fue de quimioradioterapia según esquema CROSS, aunque en algunos pacientes portadores de adenocarcinoma se utilizó solo quimioterapia. ${ }^{15,16}$

Para el registro se recurrió a la base de datos Access ${ }^{\circledR} \mathrm{e}$ informes de anatomía patológica, de los cuales se analizaron solo los ganglios mediastinales.

Análisis estadístico: los datos se reportaron como medias y desvío estándar para variables continuas paramétricas o medianas, y rango para variables continuas no paramétricas.

Las variables categóricas se reportaron como N (\%).

Para realizar la comparación del número de ganglios obtenidos entre los diferentes tipos de LN se utilizó el test de Kruskal Wallis, y para la comparar la cantidad de ganglios resecados para el AC y el CES se utilizó la prueba de U de Mann Whitney.

Se consideró en todos los casos una $p$ significativa $\leq 0,05$. Para el análisis se utilizó el programa IMB SPSS Statistics ${ }^{\circledR}$ Versión 24.0. 
Figura 2. Extensión de la linfadenectomía (decúbito prono).

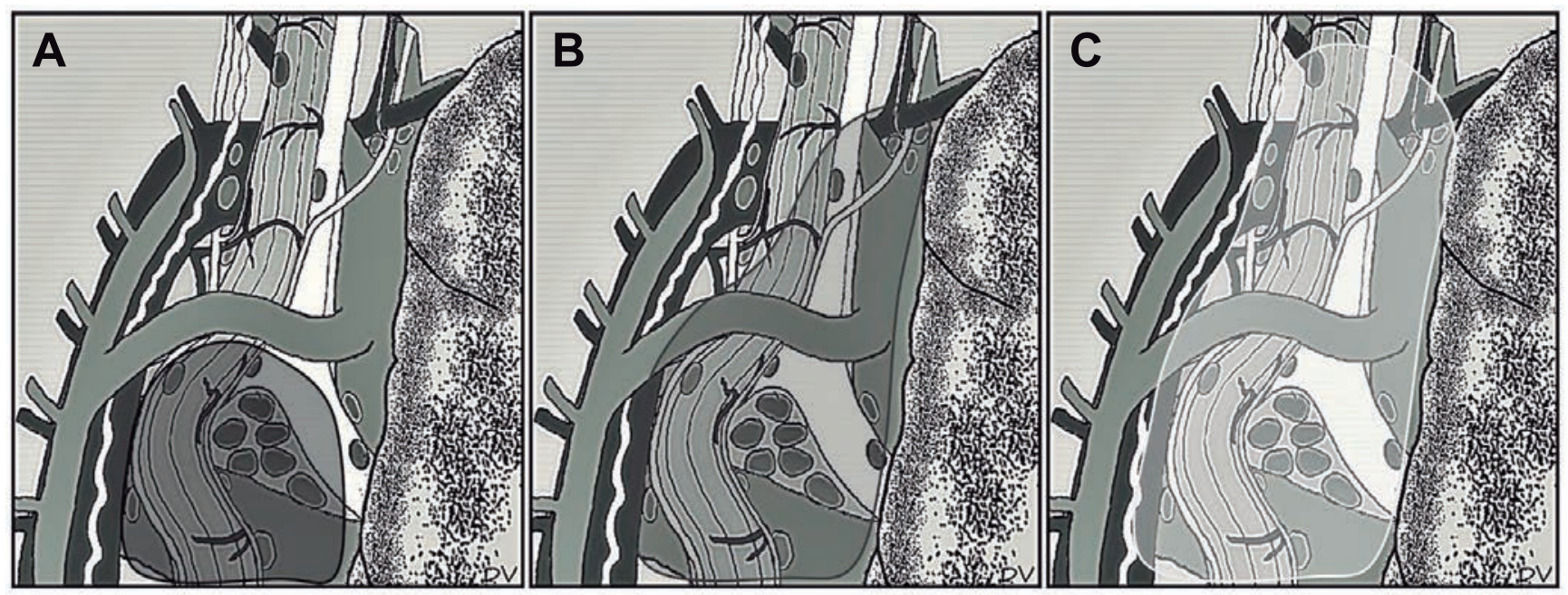

A: Estándar, B: Extendida y C: Total.

\section{Resultados}

La LN mediastinal pudo efectuarse en la mayoría de los casos, a excepción de un paciente operado por carcinoma epidermoide al inicio de la serie, que por tratarse de un estadio avanzado no fue posible lograr una cirugía R0 y, por lo tanto, la LN no se llevó cabo. Se requirió una conversión en el tiempo torácico por imposibilidad de acceder al mediastino posterior debido a importantes adherencias pleuropulmonares en un paciente con antecedentes de neumonía complicada, aunque pudo efectuarse sin inconvenientes la LN correspondiente para un adenocarcinoma.

La mediana en el número de ganglios obtenidos para el AC fue de 5,0 (3-16), mientras que para los casos de CES fue de 8,0 (4-38) ( $p=0,019)$.

El número de ganglios obtenidos para cada tipo de linfadenectomía se muestra en el Gráfico 1 y en la Tabla 2.

\section{Gráfico 1.}

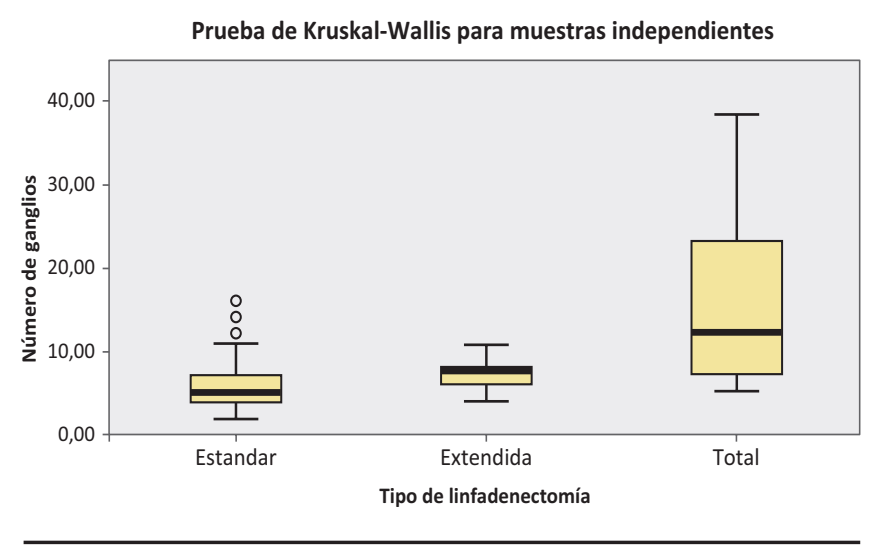

Tabla 2. Tipo de LN y número de ganglios resecados.

Tipo de linfadenectomía (N) Ganglios resecados ${ }^{*} \quad p$ valor

Estándard (34)

$5(2-16)$

Extendida (10)

$8(4-18)$

0,004

Total (6)

$12(5-38)$

En la comparación entre parejas, se evidenció la principal diferencia con una $p=0,011$ entre la linfadenectomía estándar y total.

No se evidenciaron diferencias en la comparación en el número de ganglios obtenidos entre los pacientes que no recibieron neoadyuvancia respecto de los que recibieron.

Se registraron dos complicaciones intraoperatorias: una lesión del bronquio fuente izquierdo durante la linfadenectomía subcarinal, que se pudo resolver mediante una sutura con material irreabsorbible, y una lesión del nervio recurrente derecho durante una linfadenectomía extendida. Aunque no pueda atribuirse de manera directa a la disección ganglionar, hubo un caso de neumonía grave que terminó en shock y muerte, en el cual se había realizado una linfadenectomía total.

En la figura $\mathrm{N}^{\circ} 3$ se visualiza una linfadectomia total por medio de un abordaje mininvasivo en posición prona. 
Figura 3. Linfadenectomía total.

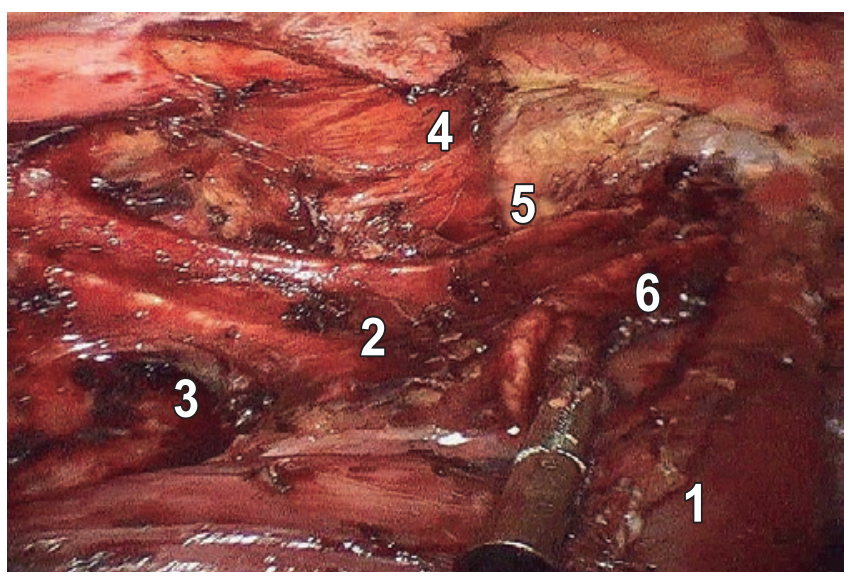

1) Esófago; 2) Bifurcación traqueal; 3) Espacio subcarinal; 4) Cayado de arteria aorta; 5) Espacio paratraqueal izquierdo; 6) Espacio paratraqueal derecho.

\section{Discusión}

Aunque no existe consenso sobre el tipo y extensión de la LN, continúa siendo un pilar fundamental en el tratamiento quirúrgico del cáncer de esófago. Si bien en la mayoría de los países occidentales para clasificar las diferentes estaciones ganglionares utilizan la propuesta por la AJCC, existe también la clasificación de la Japan Esophageal Society (JES) que se encuentra difundida en gran parte de Asia. Esta última incluye las estaciones linfáticas cervicales y, además, tiene en cuenta la topografía, a diferencia de la anterior que para la estadificación se rige solo por el número de ganglios. ${ }^{2}$ Estas diferencias en cuanto a la clasificación se traducen también en el tipo de cirugía a realizar, ya que en algunos centros asiáticos realizan cirugías por vía abierta con vaciamiento de tres campos y en Occidente mayoritariamente solo de dos campos, con cada vez más adeptos a un abordaje miniinvasivo. Por lo tanto, independientemente del tipo histológico y la prevalencia en Oriente y Occidente de uno u otro, surge la necesidad de una clasificación consensuada para disminuir las diferencias existentes en cuanto a la estrategia terapéutica y, fundamentalmente, para establecer qué tipo de LN es la más apropiada para cada caso en particular. ${ }^{16,17}$

La cirugía miniinvasiva permite una visión ampliada del mediastino y una mayor definición de la anatomía quirúrgica, hecho que promueve una mejor disección ganglionar, fundamentalmente del espacio supracarinal, en comparación con la cirugía abierta. Aunque es sabido que hay beneficios para realizar la LN del mediastino superior en decúbito lateral, también se reconoce la mejor visión que se obtiene del espacio subcarinal en posición prona. Estas pequeñas diferencias pueden solucionarse con una posición semipronado para obtener una exposición óptima de ambas regiones. ${ }^{18-23}$ La cirugía robótica ofrece resultados prometedores, debido a la posible obtención de un mayor número de ganglios resecados y con menor morbilidad en comparación con la cirugía miniinvasiva tradicional. Este hecho se debe a la visualización tridimensional y a la mayor versatilidad de los brazos robóticos para realizar movimientos muy precisos en espacios reducidos, como es en el mediastino superior, fundamentalmente para llevar a cabo linfadenectomías extendidas y totales. ${ }^{24,25}$

Los resultados de nuestro estudio demostraron la mayor obtención de un número de ganglios en aquellos pacientes portadores de carcinoma escamoso con respecto a los pacientes con adenocarcinoma. Esto es debido a la extensión de la LN realizada, extendida y total para los casos de carcinoma escamoso y solo subcarinal en los casos de adenocarcinoma. A diferencia de las mayoría de las publicaciones, en nuestra serie no se hallaron diferencias del número los ganglios resecados entre pacientes que recibieron neoadyuvancia respecto de los que no recibieron; probablemente esto se deba al tamaño de la muestra. ${ }^{26}$ Existen discrepancias acerca del número mínimo de ganglios que debieran ser resecados. Aunque es sabido que este es mayor cuando se suma un abordaje transtorácico, en muchos centros de Occidente la vía transhiatal sigue siendo de elección para el adenocarcinoma. ${ }^{14,16} \mathrm{El}$ rol actual de la quimio-radioterapia preoperatoria, plantea dudas acerca de los beneficios en la sobrevida de adicionar un abordaje transtorácico con el fin de realizar una LN mediastinal para este tipo histológico de tumor. ${ }^{2}$ Sobre la base de la difusión metastásica ganglionar de acuerdo con la ubicación del tumor, en términos generales podría resumirse que la LN más apropiada para el adenocarcinoma de tercio inferior sería la de tipo estándar, mientras que en los de ubicación en tercio medio o la presencia de adenopatías supracarinales, el vaciamiento de tipo extendido sería el conveniente. Para el carcinoma escamoso de localización distal y torácica media también el tipo de disección más apropiado sería del tipo extendido, mientras que para el de ubicación torácica superior, la LN del tipo total sería la más adecuada. ${ }^{16,27,28}$

Como complicaciones han sido descriptas lesiones de vía área, como el caso reportado en nuestra serie, lesiones vasculares principalmente de ramos traqueobronquiales o, en algunos casos, de vasos de mayor calibre como las venas pulmonares. Esta última puede producirse fundamentalmente durante la resección de los ganglios subcarinales en bloque, y más precisamente la lesión de la vena pulmonar izquierda, por encontrarse oculta detrás del 
esófago durante la disección. La linfadenectomía también se asocia a lesiones nerviosas, como de los nervios recurrentes, originando paresias o parálisis, y (en ciertas situaciones) de ramos bronquiales de los nervios vagos, ocasionando disfunciones respiratorias y neumonías, tal como podría haber sido el caso de nuestra serie. Por lo tanto, es importante ser meticuloso en la disección y reconocer las estructuras anatómicas con sus posibles variaciones con el fin de minimizar las complicaciones, principalmente cuando se realizan LN de tipo totales. ${ }^{29-31}$

Como conclusión podemos afirmar que la LN mediastinal es un procedimiento factible de realizar con un abordaje miniinvasivo en posición prona, por ofrecer una adecuada visualización de las estructuras anatómicas y la obtención de un número aceptable de ganglios. Sin embargo, está supeditada a complicaciones, que en algunos casos pueden ser graves. A su vez, independientemente del abordaje, se deberá aguardar el resultado de estudios prospectivos para evaluar los beneficios de esta en cuanto a la sobrevida, principalmente para aquellos pacientes portadores de adenocarcinoma y que han sido tratados previamente con quimio-radioterapia.

\section{Conflictos de interés. Los autores no declaran conflictos} de interés.

Cuenta con aprobación de los departamentos de Docencia e Investigación.

\section{Referencias}

1. Borggreve AS, Kingma BF, Domrachev SA, Koshkin MA, Ruurda JP, van Hillegersberg R, Takeda FR, Goense L. Surgical treatment of Esophageal cancer in the era of multimodality management. Ann N Y Acad Sci ISSN 007-89.

2. Cuesta M, Van der Peet DL, Gisbertz SS, Straatman J. Mediastinal lymphadenectomy for esophageal cancer: Diferences between two countries, Japan and Netherlands. Ann Gastreoenterol Surg 2018; 2: 176-181.

3. Van Hagen P, Hulshof MC, Van Lanschot JJ, Steyerberg EW, Van Berge Henegouwen MI, Wijnhoven BP, Richel DJ, Nieuwenhuijzen GA, Hospers GA, Bonenkamp JJ, Cuesta MA, Blaisse RJ, Busch OR, Ten Kate FJ, Creemers GJ, Punt CJ, Plukker JT, Verheul HM, Spillenaar Bilgen EJ, Van Dekken H, Van der Sangen MJ, Rozema T, Biermann K, Beukema JC, Piet AH, Van Rij CM, Reinders JG, Tilanus HW, Van der Gaast A; CROSS Group. Preoperative chemoradiotheraphy for Esophageal or junctional cancer. N Engl J Med 2012; 266: 2074-2084.

4. Siewert R, Feith M, Werner M, Stein HJ. Adenocarcinoma of the esophagogastric junction: results of surgical therapy based on anatomical/topographic classification in 1,002 consecutive patients. Ann Surg 2000; 232: 353-361.
5. D'Amico TA, Glasgow RE, Hofstetter WL, Orringer MB, Scott WJ, Varghese TK, Wright CD. Esophageal and esophago, gastric junction cancers. NCCN 2017.

6. Cuesta MA, Van der Wielen N, Weijs TJ, Bleys RL, Gisbertz SS, Van Duijvendijk P, Van Hillegersberg R, Ruurda JP, Van Berge Henegouwen MI, Straatman J, Osugi H, Van der Peet DL. Surgical anatomy of the supracarinal esophagus based on a minimally invasive approach: vascular and nervous anatomy and technical steps to resection and lymphandenectomy. Surg Endosc 2016; DOI 10.1007s00464- 016-5186-1.

7. Palanivelu C, Prakash A, Senthilkumar R, Senthilnathan P, Parthasarathi R, Rajan, Venkatachlam S. Minimally invasive esophagectomy: thoracoscopic mobilization of the esophagus and mediastinal lymphadenectomy in prone position-experience of 130 patients. J Am Coll Surg 2006; 203: 7-16.

8. Cuschieri A. Thoracoscopic subtotal oesophagectomy. Endosc Surg Allied Technol 1994; 2: 21-25.

9. Koyanagi K, Ozawa S, Tachimori Y. Minimally invasive esophagectomy performed with the patient in a prone position: a systematic review. Surg Today 2016; 46: 275-284.

10. Oshikiri T, Yasuda T, Yamamoto M, Kanaji S, Yamashita K, Matsuda T. Trainee competence in thoracoscopic esophagectomy in the prone position: evaluation using cumulative sum techniques. Langenbecks Arch Surg. 2016; 401: 797-804.

11. Li X, Lai FC, Qiu ML, Luo RG, Lin JB, Liao B. Minimally invasive esophagectomy in the lateral-prone position: experience of 226 cases. Surg Laparosc Endosc Percut Tech 2016; 26: 60-65.

12. Rice TW, Patil DT, Blackstone EH. 8th edition AJCC/UICC staging of cancers of the esophagus and esophagogastric junction: application to clinical practice. Ann Cardiothorac Surg 2017; 6 (2): 119-130.

13. Fujita H, Sueyoshi S, Tanaka T, Fujii T, Toh U, Mine T, Sasahara H, Sudo T, Matono S, Yamana H, Shirouzu K. Optimal lymphadenectomy for squamous cell carcinoma in the thoracic esophagus: comparing the short- and long-term outcome among the four types pf lymphadenectomy. World J Surg 2003; 27: 571- 579.

14. Tong D, Law S. Extended lymphadenectomy in esophageal cancer is crucial. World J Surg 2013; 27: 1751-1756.

15. Anderegg MCJ, Van der Sluis PC, Ruurda JP, Gisbertz SS, Hulshof MCCM, Van Vulpen M, Mohammed NH, Van Laarhoven HWM, Wiezer MJ, Los M, Van Berge Henegouwen MI, Van Hillegersberg R. Preoperative Chemoradiotherapy Versus Perioperative Chemotherapy for Patients With Resectable Esophageal or Gastroesophageal Junction Adenocarcinoma. Ann Surg Oncol. 2017; 24 (8): 2282-2290.

16. Koen Talsma A, Shapiro J, Looman CW, van Hagen P, Steyerberg EW, Van der Gaast A, Van Berge Henegouwen MI, Wijnhoven BP, Van Lanschot JJ; CROSS Study Group, Hulshof MC, Van Laarhoven HW, Nieuwenhuijzen GA, Hospers GA, Bonenkamp JJ, Cuesta MA, Blaisse RJ, Busch OR, Ten Kate FJ, Creemers GJ, Punt CJ, Plukker JT, Verheul HM, Van Dekken H, Van der Sangen MJ, Rozema T, Biermann K, Beukema JC, Piet AH, Van Rij CM, Reinders JG, Tilanus HW. Lymph node retrievel during esophagectomy with and without neoadyunvat chemoradiotherapy. Ann Surg 2014; 260: 786-793.

17. Shang QZ, Cheng LQ, Hu WP, Yuang Y, Cai J. There field lymph node dissection in treating the esophageal cancer. J Thotac Dis 2016; 8 (10): E1116-1149. 
18. Straatman J, Van der Wielen N, Cuesta MA, Daams F, Roig Garcia J, Bonavina. Minimally invasive versus open esophageal resection: three-year follow-up of the previously reported randomized controlled trial: the TIME trial. Ann Surg 2017; 266: 232-236.

19. Yibulayin W, Abulizi S, Lv H, Sun W. Minimally invasive oesophagectomy versus open esophagectomy for resectable esophageal cancer: a meta-analysis. World J Surg Oncol 2016; 8 (14): 304.

20. Seesing MFJ, Gisbertz SS, Goense L, Van Hillegersberg R, Kroon HM, Lagarde SM, Ruurda JP, Slaman AE, Van Berge Henegouwen MI, Wijnhoven BPL. Propensity score matched analysis of open versus minimally invasive transthoracic esophagectomy in the Netherlands. Ann Surg 2017; 266: 839-846.

21. Shen Y, Zhong M, Wu W, Wang H, Feng M, Tan L, Wang Q. The impact of tidal volume on pulmonary complications following minimally invasive esophagectomy: a randomized and controlled study. J Thorac Cardiovasc Surg 2013; 146: 1267-1273.

22. Dantoc MM, Cox MR, Eslick GD. Does minimally invasive esophagectomy (MIE) provide for comparable oncologic outcomes to open techniques? A systematic review. J Gastrointest Surg 2012; 16: 486-494.

23. Biere SS, van Berge Henegouwen MI, Maas KW, Bonavina L, Rosman C. Minimally invasive versus open oesophagectomy for patients with oesophageal cancer: a multicentre, open label, randomised controlled trial. Lancet 2012; 379: 1887-1892.

24. Chao YK, Li ZG, Wen Y-W, Kin DJ, Park SY, Chang YL, van der Sluis PC, Ruurda JP, van Hillegersberg R. Robotic-assisted esophagectomy vs video- assited thotacoscopic esophagectomy (REVATE): Study protocol for a randomized controlled trial. Trials 2019; 20 (1): 346.
25. Tagkalos E, Goense L, Hoppe-Lotichius M, Ruurda JP, Babic B, Hadzijusufovic E, Kneist W, Van der Sluis PC, Lang H, Van Hillegersberg R, Grimminger PP. Robot-assited minimally invasive esophagectomy (RAMIE) compared to conventional minimally invasive esophagectomy (MIE) for esophageal cancer: a propensity-matched analysis. Diseases of the Esophagus 2019; 0: 1-6.

26. Hagens ERC, van Berge Henegouwn MI, Cuesta M, Gisbertz SS. The extent of lymphadenectomy in Esophageal resection for cancer should be standardized. J Thorac Dis 2017; 9 (8): S713-S723.

27. Akiyama Y, Iwaya T, Endo F, Nikai H, Sato K, Baba S, Chiba T, Kimura T, Takahara T, Otsuka K, Nitta H, Mizuno M, Kimura Y, Koeda K, Sasaki A. Thoracoscopic esophagectomy with total meso- esophageal escision reduce regional lymph node recurrence. Langenbeckcks Arch Surg 2018; 403 (8): 967-975.

28. Ma H, Li Y, Ding Z, Liu X, Xu J, Qin J. The clinical significance of subcarinal lymph node dissection in the radical resection of oesophageal cancer. Interactive Cardiovascular and Thoracic Surgery $2013 ; 16: 839-843$.

29. Pennathur A, Luketich J. Complication of minimally invasive esophagectomy. In: Little AG, Merrill WH. Complications in cardiothoracic surgery (Second edition). Dayton OH. Blackwell Publishing Ltd 2010: 247-265.

30. Osugi H, Takemura M, Higashino M, Takada N, Lee S, Ueno M, Tanaka Y, Fukuhara K, Hashimoto Y, Fujiwara Y, Kinoshita $\mathrm{H}$. Learning curve of video-assisted thoracoscopic esophagectomy and extensive lymphadenectomy for squamous cell cancer of the thoracic esophagus and results. Surg Endosc 2003; 17: 515-519.

31. Dhamija A, Rosen JE, Dhamija A, Gould Rothberg BE, Kim AW, Detterbeck FC, Boffa DJ. Learning curve to lymph node resection in minimally invasive esophagectomy for cancer. Innovations (Phila) 2014; 9: 286-291. 